\title{
THE VARIABLE END POINT PROBLEM OF THE CALCULUS OF VARIATIONS INCLUDING A GENERALIZATION OF THE CLASSICAL JACOBI CONDITIONS*
}

\author{
BY
}

\author{
A. E. CURRIER
}

1. Introduction. $\dagger$ In the present paper we treat the variable end point problem of the calculus of variations in parametric form in $m$-space with end points variable on manifolds.

We set up the index form associated with an extremal segment cut transversally by two manifolds. We characterize the type numbers and nullity of this form, and apply our results to give necessary conditions and sufficient conditions for a minimum. Our characterization is partly in terms of focal

* Presented to the Society, April 9, 1932; received by the editors December 17, 1931.

$\dagger$ The reader is referred to the following articles; references in the text will be given by number.

(1) Some of the results established in this paper were announced in a note by the author, The problem of the calculus of variations in m-space with end points variable on two manifolds, Proceedings of the National Academy of Sciences, vol. 16 (1930), pp. 248-251.

(2) Morse, The foundations of the calculus of variations in the large in m-space (first paper), these Transactions, vol. 31 (1930), pp. 379-404.

(2') Morse, The order of vanishing of the determinant of a conjugate base, Proceedings of the National Academy of Sciences, vol. 17 (1931), pp. 319-320.

(3) The Jacobi conditions in their classical form for the variable end point problem in 2-space are given by Bliss, Jacobi's condition when both end points are variable, Mathematische Annalen, vol. 58 (1903), p. 70. See also Bliss, Jacobi's condition for problems of the calculus of variations in parametric form, these Transactions, vol. 17 (1916), pp. 195-206.

(4) Morse, $A$ generalization of the Sturm separation and comparison theorems in $n$-space, Mathematische Annalen, vol. 103 (1930), pp. 52-69.

(5) Bliss, The transformations of Clebsch in the calculus of variations, these Transactions, vol. 17 (1916), p. 595.

For other results on certain more general variable end point problems the reader is referred to the following articles.

Morse and Myers, The problem of Lagrange and Mayer with variable end points, Proceedings of the American Academy of Arts and Sciences, vol. 66 (1931), pp. 235-253.

Morse, Sufficient conditions in the problem of Lagrange with variable end conditions, American Journal of Mathematics, vol. 53 (1931), pp. 517-546.

Bliss, The problem of Mayer with variable end points, these Transactions, vol. 19 (1918), p. 312.

Bliss, The problem of Lagrange in the calculus of variations, American Journal of Mathematics, vol. 52 (1930), pp. 673-744.

Carathéodory, Die Methode der geodaetischen Aequidistanten und das Problem von Lagrange, Acta Mathematica, vol. 47 (1926), pp. 199-236.

Bolza, Vorlesungen ueber Variationsrechnung.

Hadamard, Leçons sur le Calcul des Variations. 
points, and partly in terms of the type numbers and nullity of a certain fundamental invariant function which we define.

The results which we obtain are new. They include a complete generalization of the classical Jacobi conditions, which so far as we know have never even been formulated for the variable end point problem in space (3), either for the problem in parametric form or for the problem in non-parametric form.

We wish to thank Professor Marston Morse for kindly reading this paper and suggesting many improvements in form of presentation which we have adopted. Our work here is closely related to Morse's paper on separation theorems (4), and depends directly on the results obtained by Morse for the one-variable-end-point problem.

\section{I}

2. The integrand. Let $R$ be an open region in the space of the variables $(z)=\left(z_{1}, \cdots, z_{m}\right)$. Let $F(z, r)=F\left(z_{1}, \cdots, z_{m}, r_{1}, \cdots, r_{m}\right)$ be a function of class $C^{4}$ for $(z)$ in $R$ and $(r)$ any set not (0). We suppose that $F$ is positively homogeneous of order one in the variables $(r)$. Let $J$ be the following integral in parametric form:

$$
J=\int F(z, \dot{z}) d t
$$

where $(\dot{z})$ stands for the set of derivatives of $(z)$ with respect to $t$.

Let $g$ be an extremal segment lying in $R$. We suppose that $g$ is an ordinary curve of class $C^{\prime \prime}$. Let $F_{i}$ and $F_{i j}$ denote the first and second partial derivatives of $F$ with respect to the $(r)$ 's in the usual way. We assume that $F$ is positively regular along $g$, that is, we suppose that*

$$
F_{i j} u_{i} u_{j}>0
$$

$$
(i, j=1, \cdots, m) \text {, }
$$

where the arguments of the partial derivatives of $F$ are $(z, \dot{z})$ taken along $g$, and $(u)$ is any set not $(0)$ nor proportional to $(\dot{z})$.

We assume that $F(z, \dot{z})$ is positive for $(z, \dot{z})$ taken along $g$. This assumption is a matter of convenience, and is not an essential restriction on the problem.

3. Admissible fields of extremals. Let $H$ be an $(n=m-1)$-parameter family of extremals whose equations are given parametrically in the form

$$
z_{i}=z_{i}(t, \sigma)=z_{i}\left(t, \sigma_{1}, \cdots, \sigma_{n}\right) \quad(i=1, \cdots, m ; n=m-1)
$$

where the above functions together with their first partial derivatives with

* We adopt the convention here and elsewhere that the repetition of a subscript denotes summation with respect to that subscript. 
respect to $t$ are of class $C^{\prime \prime}$ for $(\sigma)$ near $\left(\sigma^{0}\right)$ and $t$ on the closed segment $(a, b)$ of the $t$-axis.

The family $H$ will be said to constitute an admissible field if the following four conditions are satisfied.

(i) The extremal $g$ is a member of $H$, and for $(\sigma)=\left(\sigma^{0}\right)$ (3.1) give the equations of $g$ in terms of arc length $t$ as parameter.

(ii) For $(\sigma)$ constant near $\left(\sigma^{0}\right),(3.1)$ give the equations of an extremal $\gamma$ of the family $H$, and the parameter $t$ is arc length along $\gamma$.

(iii) The Jacobian of the functions (3.1) with respect to the parameters $(t, \sigma)$ evaluated for $(\sigma)=\left(\sigma^{0}\right)$ vanishes, if at all, for isolated values of $t$ on the closed segment $(a, b)$ of the $t$-axis.

(iv) The family $H$ is a Mayer field, that is, the Hilbert invariant integral exists and is independent of the path for all paths lying in the field.*

4. Focal points and transverse manifolds. Let $H$ be an admissible field of extremals as defined in the preceding section. Let the equations of $B$ be given in the form (3.1).

The points of $g$ corresponding to values of the parameter $t$ for which the Jacobiant of the functions (3.1) vanishes are said to be the focal points (on $g$ ) of the field $H$. The order of a focal point is defined as the nullity of the Jacobian matrix at the corresponding point. $\ddagger$

Let $(\bar{z})$ be a point of $g$ not a focal point of the field $H$. The set of first partial derivatives of the functions (3.1) with respect to $t$ can be expressed as a set of functions of $(z)$ for $(z)$ near $(\bar{z})$. This set of functions, which we denote by $r(z)$, is said to be the set of direction cosine $\$$ functions of the field $H$. The particular set of values that $\boldsymbol{r}(z)$ take on for a fixed value of $(z)$ is said to be the set of direction cosines of the field $H$ at the point $(z)$. The set $r(\bar{z})$ is said to be the set of direction cosines of $g$ at $(\bar{z})$, and will be denoted as the set $(\bar{r})$.

Let $V(z)$ be the Hilbert integral associated with the field $H$. The function $V(z)$ is uniquely defined (up to an additive constant) for $(z)$ near $(\bar{z})$, where $(\bar{z})$ is any point of $g$ not a focal point of $H$. As is well known, the Hilbert integral has the following explicit form as a line integral:

$$
V(z)=\int F_{i} d z_{i} \quad(i=1, \cdots, m),
$$

* Cf. Bliss (5), loc. cit. The Hilbert integral can be expressed either as a function of the parameters $(t, \sigma)$ or as a function of the coördinates $(z)$ of points in the space $(z)$. When expressed as a function of the parameters the Hilbert integral is uniquely defined for $(\sigma)$ near $\left(\sigma^{0}\right)$ and $t$ on the closed segment $(a, b)$ of the $t$-axis. When expressed as a function of $(z)$ the Hilbert integral is uniquely defined for all points near points of $g$ not focal points of the field $H$.

$\dagger$ That is, the Jacobian of (3.1) with respect to the $m$ variables $(t, \sigma)$, evaluated for $(\sigma)=\left(\sigma^{0}\right)$.

$\ddagger$ Cf. Morse (2), loc. cit., pp. 383-384, also p. 397; also Morse (2'), loc. cit.

$\S$ We recall that the parameter $t$ gives arc length along the extremals of the field $H$. 
where the arguments $(r)$ of the partial derivatives of $F$ are taken as the set of direction cosine functions $\boldsymbol{r}(\boldsymbol{z})$.

We see from (4.1) that the first partial derivatives of the function $V(z)$ are equal respectively to the partial derivatives $F_{i}[z, r(z)](i=1, \cdots, m)$. For $(z)=(\bar{z})$ these partial derivatives do not vanish simultaneously, as is well known, since the integrand $F$ is positive along $g$. The manifold $M$, whose equation may be written as follows,

$$
V(z)-V(\bar{z})=0,
$$

is said to be the transverse manifold of the field $H$ at the point $(\bar{z})$ of $g$. Because of the fact that the first partial derivatives of $V(z)$ do not vanish simultaneously for $(z)=(\bar{z})$, equation (4.2) can be solved for one of the $(z)$ 's in terms of the remaining $n=m-1$ of the (z)'s as independent variables. This fact is well known, and its proof follows at once from well known implicit function theorems.

5. Admissible broken extremals. Let $H$ and $H^{\prime}$ be two admissible fields of extremals. Let $M$ be a transverse manifold of the field $H$, where $M$ cuts $g$ at some point not a focal point of $H$. Let $M^{\prime}$ be a transverse manifold of $H^{\prime}$ cutting $g$ at some point not a focal point of $H^{\prime}$. We assume that the positive sense on $g$ is the sense from $M$ to $M^{\prime}$.

Let

$$
M, M^{1}, \cdots, M^{\alpha}, M^{\prime}
$$

be $\alpha+2$ manifolds, of which the first and the last are defined above. Let the manifolds (5.1) cut $g$ at successive points, and let them be so close together that there are no pairs of conjugate points on any of the closed segments of $g$ between successive manifolds.

We assume that the interior manifolds, that is, the manifolds $\left(M^{1}, \cdots\right.$, $\left.M^{\alpha}\right)$, cut $g$ at ordinary points, that is, at points which are not focal points of $H$ or of $H^{\prime}$.

We assume that the equations of the manifold $M^{k}$ are given parametrically in terms of $n=m-1$ of the (z)'s as parameters, and that the equations are of class $C^{\prime \prime}$. We assume that $M^{k}$ is not tangent to $g$.

Let

$$
P, P^{1}, \cdots, P^{\alpha}, P^{\prime}
$$

be $\alpha+2$ points neighboring $g$ and lying on the manifolds (5.1) respectively. The points (5.2) can be joined by unique successive extremal segments neighboring $g$ and forming a broken extremal $E$, said to be an admissible broken extremal. 
Let $(u)=\left(u_{1}, \cdots, u_{\mu}\right)$ be $\mu=(\alpha+2) n$ variables. We assume that the set (u) consists of $\alpha+2$ successive subsets, and that these subsets are sets of parametric coördinates on the successive manifolds (5.1). Let $\left(u^{0}\right)$ be the set of parameters $(u)$ which corresponds to the set of points in which the manifolds (5.1) are cut by $g$. Let $(u)$ be the set of parameters $(u)$ which corresponds to the set of points (5.2). We shall say that the set $(u)$ is the set of parametric coördinates of the admissible broken extremal $E$.

6 . The index form associated with two transverse manifolds. Let $H$ and $H^{\prime}$ be two admissible fields of extremals, and let $M$ and $M^{\prime}$ be two transverse manifolds of $H$ and $H^{\prime}$ respectively.

Let $E$ be an admissible broken extremal as defined in the preceding section, and let $(u)$ be the parametric coördinates of $E$.

The integral (2.1) taken along $E$ is a function of $(u)$ which we denote by $J(u)$. For $(u)=\left(u^{0}\right), E$ becomes the segment of $g$ between $M$ and $M^{\prime}$. The function $J(u)$ has a critical point for $(u)=\left(u^{0}\right)$.

The index form $Q(u)$ is defined as follows:

$$
Q(u)=J_{i j}^{0} u_{i} u_{j} \quad(i, j=1, \cdots, \mu),
$$

where the partial derivatives of $J$ are denoted in the usual way, and the superscripts zero indicate that these partial derivatives are to be evaluated for $(u)=\left(u^{0}\right)$.

7. The fundamental lemmas on the index form. Let $(v)=\left(v_{1}, \cdots, v_{n}\right)$ be the set of parameters on the manifold $M^{k}$. The set $(v)$ is a subset of the variables $(u)$ which have been defined as the parametric coördinates of admissible broken extremals. Let $(x)$ be the complementary subset. We now denote the variables $(u)$ interchangeably as $(u)$ and as $(x, v)$.

Let $E$ be an admissible broken extremal which has no corners except on the manifold $M^{k}$. Let the first segment of $E$ between $M$ and $M^{k}$ be a member of the field $H$, and let the second segment of $E$ from $M^{k}$ to $M^{\prime}$ be a member of the field $H^{\prime}$. The broken extremal $E$ is uniquely determined by the corner point on $M^{k}$. Let $(x, v)=\left(x^{*}, v\right)$ be the parametric coördinates of $E$. We readily verify the fact that the elements of the set $\left(x^{*}\right)$ can be expressed uniquely as functions of class $C^{\prime \prime}$ of the variables $(v)$. This follows from the fact that the manifold $M^{k}$ does not cut $g$ in a focal point of $H$ or of $H^{\prime}$. The proof involves well known implicit function theorems and can be left to the reader.

The first variation of the integral (2.1) vanishes along the broken extremal $E$ defined above, except possibly at the corner of $M^{k}$. Hence the following first partial derivatives are zero:

$$
J_{x_{i}}\left(x^{*}, v\right)=0,
$$


where the subscript ( $i$ ) runs through the set of all subscripts associated with the set of variables $(x)$, and $(v)$ is any set near $\left(v^{0}\right)$.

We replace the variables $(v)$ on the left of $(7.1)$ by the variables $\left(v^{0}+e v\right)$, thus obtaining an identity in $e$ for $e$ near zero. We differentiate the left hand side of this identity once with respect to $e$ and set $e=0$. We obtain the result

$$
J_{x_{i} x_{j}}^{0} \frac{\partial x_{j}^{*}}{\partial v_{k}} v_{k}+J_{x_{i} v_{k} v_{k}}^{0}=0,
$$

where the partial derivatives of the functions $\left(x^{*}\right)$ are to be evaluated for $(v)=\left(v^{0}\right)$, and the remaining notation is self explanatory.

Let $\left(x^{\prime}\right)$ denote the linear combination of $(v)$ which occurs on the left of (7.2). That is, let $\left(x^{\prime}\right)$ be the set

$$
x_{j}^{\prime}=\frac{\partial x_{j}^{*}}{\partial v_{k}} v_{k},
$$

where the partial derivatives involved are evaluated for $(v)=\left(v^{0}\right)$ and where the values through which the various subscripts run are indicated by the variables to which they are attached.

We now evaluate the index form on the sum of the sets $\left(u^{1}\right)=(x, 0)$ and $\left(u^{2}\right)=\left(x^{\prime}, v\right)$. We use a well known formula $\nmid$ which is

$$
Q\left(u^{1}+u^{2}\right)=Q\left(u^{1}\right)+Q\left(u^{2}\right)+2 B Q\left(u^{1}, u^{2}\right),
$$

where $B Q$ denotes the bilinear form whose matrix is the same as the matrix of $Q$.

We see from (7.2) that the bilinear form $B Q$ is identically zero on the variables $\left(u^{1}\right)=(x, 0),\left(u^{2}\right)=\left(x^{\prime}, v\right)$. The quadratic form $Q\left(u^{1}\right)=Q(x, 0)$ is a form on the variables $(x)$ alone. The form $Q\left(u^{2}\right)=Q\left(x^{\prime}, v\right)$ is a form on the variables $(v)$ alone, since $\left(x^{\prime}\right)$ depends linearly on the $(v)$ 's. We have thus proved the following lemma.

LeMma 1. The index form $Q(u)$ evaluated on the variables $(u)=\left(x+x^{\prime}, v\right)$, where $(x)$ and (v) are arbitrary and $\left(x^{\prime}\right)$ depends linearly on the (v)'s as in (7.3), is identically equal to the sum of a form on the variables $(x)$ alone plus a form on the variables $(v)$ alone, viz. to $Q(x, 0)+Q\left(x^{\prime}, v\right)$.

A corollary of Lemma 1 may be stated at once as follows.

LEMMa 2. The negative type number of the index form $Q(u)$ is equal to the sum of the negative type numbers of $Q(x, 0)$ and $Q\left(x^{\prime}, v\right)$, and the nullity of the index form is equal to the sum of the nullities of the forms $Q(x, 0)$ and $Q\left(x^{\prime}, v\right)$.

† Bocher, Introduction to Higher Algebra, New York, 1907, p. 119. 
8. The quadratic form $Q\left(x^{\prime}, v\right)$. Let $E$ be the broken extremal defined in the preceding section, which has one corner (on $M^{k}$ ) and whose first and second segments are members of the fields $H$ and $H^{\prime}$ respectively.

The integral (2.1) taken along $E$ can be expressed in terms of the Hilbert integrals. Let $V(z)$ and $V^{\prime}(z)$ be the Hilbert integrals associated with the fields $H$ and $H^{\prime}$ respectively. Then

$$
J\left(x^{*}, v\right)=V(z)-V^{\prime}(z)+\text { const. }
$$

where $\left(x^{*}, v\right)$ are the parametric coördinates of $E$, and where it is understood that the Hilbert integrals $V(z)$ and $V^{\prime}(z)$ are to be evaluated for $(z)$ taken at the point (v) of the manifold $M^{k}$.

Let the equations of the manifold $M^{k}$ be given in terms of the parameters (v) as follows:

$$
z_{i}=z_{i}(v)=z_{i}\left(v_{1}, \cdots, v_{n}\right) \quad(i=1, \cdots, m) .
$$

Let $d_{i j}$ be the value of the first partial derivative of the function $z_{i}(v)$ with respect to $v_{j}$ for $(v)=\left(v^{0}\right)$.

We now replace the variables $(v)$ on the left of (8.1) by the variables $\left(v^{0}+e v\right)$, remembering not only that the set $(v)$ occurs in the arguments of $J\left(x^{*}, v\right)$ as indicated explicitly but that also each element of the set $\left(x^{*}\right)$ is a function of $(v)$ as explained in the preceding section. We replace the variables (v) on the right of (8.2) by the variables $\left(v^{0}+e v\right)$, and we assume that the resulting functions of $e$ are substituted for the arguments $(z)$ of the Hilbert integrals which occur on the right of (8.1). Equation (8.1) then becomes an identity in $e$ for $e$ near zero. We differentiate each side of this identity twice with respect to $e$ and set $e=0$. On the left we obtain the form $Q\left(x^{\prime}, v\right)$, and on the right we obtain an explicit expression for this form. We thus establish the identity

$$
Q\left(x^{\prime}, v\right)=\left(V_{h k}-V_{h k}^{\prime}\right) d_{h i} d_{k j} v_{i} v_{j} \quad(h, k=1, \cdots, m ; i, j=1, \cdots, n),
$$

where the arguments of the partial derivatives of the Hilbert integrals are the coördinates $(\bar{z})$ of the point in which $M^{k}$ cuts $g$.

We use the explicit formula (4.1) for the Hilbert integrals. We perform the differentiation indicated on the right of (8.3). We obtain the further result

$$
Q\left(x^{\prime}, v\right)=F_{s h}\left(\frac{\partial r_{h}}{\partial z_{k}}-\frac{\partial r_{h}^{\prime}}{\partial z_{k}}\right) d_{k i} d_{s j} v_{i} v_{j}(h, k, s=1, \cdots, m ; i, j=1, \cdots, n),
$$

where $r(z)$ and $r^{\prime}(z)$ are the direction cosine functions of the fields $H$ and $H^{\prime}$ respectively. The arguments of the partial derivatives of $F$ are $(\bar{z}, \bar{r})$, where $(\bar{z})$ is the point in which $M^{k}$ cuts $g$ and $(\bar{r})$ is the set of direction cosines of $g$ 
at this point. The partial derivatives of the direction cosine functions are evaluated for $(z)=(\bar{z})$.

By hypothesis ( $\$ 5)$ the parameters (v) are $n=m-1$ of the (z)'s. Hence the matrix of partial derivatives of the functions (8.2) is of rank $n$, that is, $d$ is of rank $n$. By hypothesis ( $\$ 5)$ the manifold $M^{k}$ is not tangent to $g$. Hence the set $(\bar{r})$ is linearly independent of the columns of $d$. Hence the matrix $\|\bar{r} d\|$ is of rank $m$.

Now let $v_{0}$ be an arbitrary variable. We define a set of variables $(w)=\left(w_{1}\right.$, $\left.\cdots, w_{m}\right)$ in terms of the variables $\left(v_{0}, v_{1}, \cdots, v_{n}\right)$ as follows:

$$
w_{i}=\bar{r}_{i} v_{0}+d_{i j} v_{j} \quad(i=1, \cdots, m ; j=1, \cdots, n ; n=m-1) .
$$

We make use of the well known identity $F_{i j}(z, r) r_{j}=0(i, j=1, \cdots, m)$. We find that

$$
Q\left(x^{\prime}, v\right)=F_{s h}\left(\frac{\partial r_{h}}{\partial z_{k}}-\frac{\partial r_{h}^{\prime}}{\partial z_{k}}\right) w_{s} w_{k} \quad(s, h, k=1, \cdots, m),
$$

where $(w)$ is given by $(8.5)$, with $v_{0}$ arbitrary, and $Q\left(x^{\prime}, v\right)$ is the quadratic form which occurs on the left of (8.4).

The form on the left of (8.6) is a quadratic form in $m-1$ variables (v) (the set $\left(x^{\prime}\right)$ depending linearly on $(v)$ ). The form on the right of (8.6) is a form on $m$ variables. The two forms are related by the non-singular collineation (8.5). Hence the following lemma is true.

LEMMA 1. The negative type number of the form $Q\left(x^{\prime}, v\right)$ is equal to the negative type number of the form on the right of (8.6), where $(w)$ is an arbitrary set of variables. The nullity of $Q\left(x^{\prime}, v\right)$ is one less than the nullity of the form on the right of (8.6).

The form on the right of (8.6) does not depend upon the manifold $M^{k}$. Hence the following lemma is true.

LeMMa 2. The negative type number and nullity of $Q\left(x^{\prime}, v\right)$ are independent of the manifold $M^{k}$.

The form $Q(x, 0)$ referred to in Lemma $2, \S 7$, is from its definition independent of the manifold $M^{k}$. Hence by Lemma 2, $\$ 7$, and Lemma 2 of this section, the negative type number and nullity of the index form $Q(u)$ are independent of the manifold $M^{k}$. This result applies to each of the manifolds $\left(M^{1}, \cdots, M^{\alpha}\right)$ defined in $\S 5$. Hence the following lemma is true.

LEMMA 3. Let the manifolds $\left(M^{1}, \cdots, M^{\alpha}\right)$ be replaced by another set of admissible manifolds cutting $g$ at the same successive points. Then the negative type number and nullity of the index form $Q(u)$ remain unchanged. 
9. The quadratic form $Q(x, 0)$. The quadratic form $Q(x, 0)$ is the index form $Q(u)$ evaluated on the set $(u)=(x, 0)$. That is, $Q(x, 0)$ is obtained by setting the variables $(v)=(0)$ in the index form. Let $E$ be an admissible broken extremal passing through the point $(\bar{z})$ in which $M^{k}$ cuts $g$. The subset (v) of the set of parametric coördinates of $E$ is equal to $\left(v^{0}\right)$. The parametric coördinates of $E$ can be written as follows: $(u)=\left(x^{0}+e x, v^{0}\right)$. The quadratic form $Q(x, 0)$ is then equal to the expression on the right of the following equation:

$$
Q(x, 0)=\frac{d^{2}}{d e^{2}} J\left(x^{0}+e x, v^{0}\right),
$$

where the second derivative on the right is evaluated for $e=0$.

The function $J\left(x^{0}+e x, v^{0}\right)$ whose second derivative occurs on the right of (9.1) is essentially the sum of two functions, viz. the integral (2.1) taken along the broken extremal $E$ from $M$ to the point $(\bar{z})$ plus the same integral taken along $E$ from $(\bar{z})$ to $M^{\prime}$. These two functions are independent of each other. We readily see that the form $Q(x, 0)$ is equal to the sum of two quadratic forms, the first form being on the subset of the variables $(x)$ which corresponds to the parameters on the manifolds $M, M^{1}, \cdots, M^{k-1}$. The other form is on the subset of the variables $(x)$ which corresponds to the parameters on the manifolds $M^{k+1}, \cdots, M^{\alpha} M^{\prime}$. These two subsets are non-overlapping. The quadratic form on the first subset of the variables $(x)$ is an index form associated with the problem of the calculus of variations in which one end point varies on the manifold $M$ and the other end point is the fixed point $(\bar{z})$. Its negative type number is equal to the sum of the orders of the focal points of the field $H$ on the segment of $g$ between $M$ and ( $\bar{z})$, and its nuility is zero.* Similarly for the form on the complementary set of variables. Thus the following lemma is true.

LEMMA 1. The nullity of the form $Q(x, 0)$ is zero, and the negative type number of the form $Q(x, 0)$ is equal to the sum of the orders of the focal points of the field $H$ on the open $\dagger$ segment of $g$ between $M$ and $(\bar{z})$, plus the sum of the orders of the focal points of the field $H^{\prime}$ on the open segment of $g$ between $(\bar{z})$ and $M^{\prime}$.

10. The fundamental invariant function $I(z, w)$. The invariant function $I(z, w)$ is defined in terms of the Hilbert integrals as follows:

$$
I(z, w)=\left(V_{i j}-V_{i j}^{\prime}\right) w_{i} w_{j} \quad(i, j=1, \cdots, m)
$$

* Morse (2), loc. cit., p. 399, Theorems 3 and 4. In order to apply the results of Morse's Theorems 3 and 4 we require merely the result announced by our Lemma $3, \$ 8$. In applying Theorem 3 we remember that the point $(\bar{z})$ is not a focal point of the field $H$ (or $H^{\prime}$ ).

$\dagger$ This segment can be open or closed, since its end points are not focal points of the field $H$. 
where $V(z)$ and $V^{\prime}(z)$ are the Hilbert integrals associated with the admissible fields $H$ and $H^{\prime}$ respectively, where $(z)$ is any point for which both $V(z)$ and $V^{\prime}(z)$ are defined, and $(w)$ is any set.

We now state two lemmas concerning the invariant function.

LEMMA 1. Associated with each point (z) for which the invariant function $I(z, w)$ exists there are two absolute numerical invariants, viz. the negative type number and the nullity of $I(z, w)$.

LEMMA 2. The negative type number of the quadratic form $Q\left(x^{\prime}, v\right)$ is equal to the negative type number of the invariant function $I(z, w)$ evaluated for $(z)$ $=(\bar{z})$, where $(\bar{z})$ is the point in which $M^{k}$ cuts $g$. The nullity of $Q\left(x^{\prime}, v\right)$ is one less than the nullity of $I(\bar{z}, w)$.

Lemma 1 follows at once from the definition of the invariant function. Lemma 2 follows from Lemma $1, \S 8$.

11. The type number and nullity of the index form. Let $q$ be the sum of the orders of the focal points of the field $H$ on the open segment of $g$ between $M$ and $(\bar{z})$, where $(\bar{z})$ is any point of the open segment of $g$ between $M$ and $M^{\prime}$ excepting focal points of $H$ and $H^{\prime}$. Let $q^{\prime}$ be the sum of the orders of the focal points of $H^{\prime}$ on the open segment of $g$ between $(\bar{z})$ and $M^{\prime}$.

Let $N$ and $h$ be respectively the negative type number and nullity of the invariant function evaluated for $(z)=(\bar{z})$.

THEOREM 1. The negative type number $\left(N^{0}\right)$, and the negative type number plus the nullity $\left(N^{0}+h^{0}\right)$, of the index form $Q(u)$ are given by the equations

$$
N^{0}=q+q^{\prime}+N, N^{0}+h^{0}=q+q^{\prime}+N+h-1 \text {. }
$$

We can choose the manifolds (5.1) so that one of them, say the manifold $M^{k}$, cuts $g$ at the point $(\bar{z})$. Then Theorem 1 follows directly from the following lemmas, Lemma $2, \S 7$, Lemma $1, \$ 9$, and Lemma $2, \$ 10$.

12. Conditions for a minimum. By a regular integral $J$ we mean an integral of the form (2.1) where the hypotheses of $\$ 2$ are all satisfied. We can use the results of Theorem 1 to give necessary conditions and sufficient conditions for a minimum. It is necessary that the index form $Q(u)$ be positive in order that the regular integral $J$ take on a minimum along the segment of $g$ between $M$ and $M^{\prime}$. It is sufficient for a weak proper relative minimum that the index form $Q(u)$ be positive definite. The index form is positive if and only if its negative type number is zero, and positive definite if and only if the sum of its negative type number plus its nullity is zero.

We state the results in the form of theorems as follows. 
THEOREM 2. In order that the regular integral $J$ take on a minimum along $g$ it is necessary that there be no focal points of $H$ or of $H^{\prime}$ on the open segment of $g$ between $M$ and $M^{\prime}$, and that the invariant function $I(z, w)$ satisfy the inequality

$$
I(z, w) \geqq 0
$$

for (z) taken along the open segment of $g$ between $M$ and $M^{\prime}$, where (w) is any set. The inequality (12.1) is merely the condition that the negative type number $N$ of the invariant function $I(z, w)$ be zero, for all points $(z)$ of the open segment of $g$ between $M$ and $M^{\prime}$.

THEOREM 3. In order that the regular integral $J$ take on a weak proper relative minimum along $g$ it is sufficient that there be no focal points of $\boldsymbol{H}$ or of $\boldsymbol{H}^{\prime}$ on the open segment of $g$ between $M$ and $M^{\prime}$, and that there exist at least one point $(\bar{z})$ of the open segment of $g$ between $M$ and $M^{\prime}$ such that the following inequality is satisfied for $(z)=(\bar{z})$ :

$$
I(\bar{z}, w)>0,
$$

where $I(\bar{z}, w)$ is the invariant function evaluated for $(z)=(\bar{z})$, and $(w)$ is any set not $(0)$ nor proportional to $(\bar{r})$, where $(\bar{r})$ is the set of direction cosines of $g$ at $(\bar{z})$.

We see that (12.2) is a necessary and sufficient condition in order that $N+h-1=0$, where $N$ is the negative type number of $I(\bar{z}, w)$, and $h$ is the nullity of the same form. We see that if the conditions of Theorem 3 are satisfied the expression on the right of the second equation (11.1) will be zero, which is necessary and sufficient in order that the index form $Q(u)$ be positive definite.

THEOREM 4. Let $J$ be a regular integral, and let the Weierstrass $E$-function $E(z, r, \sigma)$ be positive for $(z, r)$ taken along the closed segment of $g$ between $M$ and $M^{\prime}$, where $(\sigma)$ is any set not $(0)$ nor proportional to $(r)$. Let there be no focal points of $H$ or of $H^{\prime}$ on the open segment of $g$ between $M$ and $M^{\prime}$. Let there exist at least one point $(\bar{z})$ of the open segment of $g$ between $M$ and $M^{\prime}$ for which the inequality (12.2) is satisfied. Then the integral $J$ takes on a strong proper relative minimum along $g$.

THEOREM 5. The necessary conditions of Theorem 2 are not only necessary but sufficient in order that the index form $Q(u)$ be positive, and the sufficient conditions of Theorem 3 are not only sufficient but necessary in order that the index form be positive definite.

Theorem 5 shows that the conditions of Theorems 3 and 4 are as close to being both necessary and sufficient as it is possible to give without making a study of higher variations than the second variation of the integral $J$. 
II. The generalized Jacobi conditions

13. A separation theorem. Let $\bar{g}$ be any closed segment of $g$ whose end points $\left(z^{1}\right)$ and $\left(z^{2}\right)$ are not focal points of $H$ or of $H^{\prime}$. We assume that the positive sense on $g$ is the sense from $\left(z^{1}\right)$ to $\left(z^{2}\right)$.

Let $k$ and $k^{\prime}$ be respectively the sums of the orders of the focal points of $H$ and $H^{\prime}$ on the closed segment $\bar{g}$ of $g$.

Let $I(z, w)$ be the invariant function. Let $N^{1}$ and $h^{1}$ be respectively the negative type number and nullity of $I\left(z^{1}, w\right)$, and let $N^{2}$ and $h^{2}$ be respectively the negative type number and nullity of $I\left(z^{2}, w\right)$.

THEOREM 6. The number of focal points of $B$ and $H^{\prime}$ on the closed segment $\bar{g}$ of $g$, and the type numbers of the invariant function $I(z, w)$ at the ends of this segment are related by the following identities:*

$$
N^{1}+k^{\prime}=N^{2}+k, h^{1}=h^{2} \text {. }
$$

We can assume without loss of generality that the closed segment $\bar{g}$ of $g$ lies on the open segment of $g$ between $M$ and $M^{\prime}$. If this condition is not satisfied we select new transverse manifolds, $M, M^{\prime}$, which do enclose the segment $\bar{g}$ of $g$ on the open segment between them.

Now let $q$ be the sum of the orders of the focal points of $H$ on the open segment of $g$ between $M$ and $\left(z^{1}\right)$, and let $q^{\prime}$ be the sum of the orders of the focal points of $H^{\prime}$ on the open segment of $g$ between $\left(z^{1}\right)$ and $M^{\prime}$. By Theorem 1, (11.1), the negative type number of the index form $Q(u)$ is given by the equation

$$
N^{0}=q+q^{\prime}+N^{1}
$$

where $N^{1}$ is the negative type number of $I\left(z^{1}, w\right)$.

We can also apply Theorem $1,(11.1)$, to the point $\left(z^{2}\right)$ of the open segment of $g$ between $M$ and $M^{\prime}$. We obtain the further result

$$
N^{0}=q+k+q^{\prime}-k^{\prime}+N^{2},
$$

where $N^{2}$ is the negative type number of $I\left(z^{2}, w\right)$, where $q+k$ is the sum of the orders of the focal points of $H$ on the open segment $\dagger$ of $g$ between $M$ and $\left(z^{2}\right)$, and where $q^{\prime}-k^{\prime}$ is the sum of the orders of the focal points of $H^{\prime}$ on the open segment $\dagger$ of $g$ between $\left(z^{2}\right)$ and $M^{\prime}$.

The right hand sides of (13.2) and (13.3) are different expressions for the

* Cf. Morse (4), loc. cit., p. 64, Theorem 6.

$\dagger$ These segments of $g$ can be taken as either open or closed, since in any case the end points of the segnent involved are not focal points of the field of extremals involved. 
same thing, i.e. for $N^{0}$. If we set them equal to each other and cancel out $q$ and $q^{\prime}$ on each side we obtain the first equation (13.1).

The nullity of the index form $Q(u)$ is always one less than the nullity of $I(z, w)$, for $(z)$ any ordinary point of $\bar{g}$. This follows from Theorem 1 . Hence the nullity of $I\left(z^{1}, w\right)$ is the same as the nullity of $I\left(z^{2}, w\right)$. Thus the second equation (13.1) is true.

14. The Jacobi conditions. Let $M$ and $M^{\prime}$ be two transverse manifolds of the admissible fields $H$ and $H^{\prime}$ respectively. Let the positive sense on $g$ be the sense from $M$ to $M^{\prime}$, and let $Q(u)$ be the index form associated with the manifolds $M$ and $M^{\prime}$.

Let $(\bar{z})$ be a point of $g$ which lies in the positive direction from $M^{\prime}$, that is, $(\bar{z})$ does not lie on the open segment of $g$ between $M$ and $M^{\prime}$. Let $(\bar{z})$ be an ordinary point of $g$.

Let $q$ be the sum of the orders of the focal points of $H$ on the open segment of $g$ between $M$ and $(\bar{z})$. Let $q^{\prime}$ be the sum of the orders of the focal points of $H^{\prime}$ on the open segment of $g$ between $M^{\prime}$ and $(\bar{z})$.

Let $N$ and $h$ be respectively the negative type number and nullity of $I(\bar{z}, w)$.

THEOREM 7. The negative type number $\left(N^{0}\right)$, and the negative type number plus the nullity $\left(N^{0}+h^{0}\right)$, of the index form $Q(u)$ are given by the equations

$$
N^{0}=q-q^{\prime}+N, \quad N^{0}+h^{0}=q-q^{\prime}+N+h-1 .
$$

Theorem 7 follows at once if we combine the results of Theorem 1 with the results of Theorem 6 , as we now prove.

Let $\left(z^{1}\right)$ be an ordinary point of the open segment of $g$ between $M$ and $M^{\prime}$, and let $\left(z^{2}\right)$ be an ordinary point of $g$ which lies in the positive direction from $M^{\prime}$. Let $\bar{g}$ be the closed segment of $g$ between $\left(z^{1}\right)$ and $\left(z^{2}\right)$.

Let $q_{1}$ be the sum of the orders of the focal points of $H$ between $M$ and $\left(z^{1}\right)$, and let $q_{1}^{\prime}$ be the sum of the orders of the focal points of $H^{\prime}$ between $\left(z^{1}\right)$ and $M^{\prime}$. Let $N^{1}$ be the negative type number of $I\left(z^{1}, w\right)$. Then by Theorem 1 the negative type number $N^{0}$ of the index form is given by the equation

$$
N^{0}=q_{1}+q_{1}^{\prime}+N^{1} .
$$

Now let $N^{2}$ be the negative type number of $I\left(z^{2}, w\right)$, and let $k$ and $k^{\prime}$ be respectively the sums of the orders of the focal points of $H$ and $H^{\prime}$ on the segment $\bar{g}$ of $g$ which lies between $\left(z^{1}\right)$ and $\left(z^{2}\right)$. By Theorem $6, N^{1}=N^{2}+k$ $-k^{\prime}$. We substitute the expression $N^{2}+k-k^{\prime}$ for $N^{1}$ in equation (14.2), thus establishing the result announced by the first equation (14.1). The second equation (14.1) can now be readily established. 
15. The generalization of the classical Jacobi conditions. ${ }^{*}$ Let the equations of $g$ be given parametrically in terms of $\tau$ as parameter, for $\tau$ on the closed segment $(a, b)$ of the $\tau$-axis.

Let $t$ be the $\tau$-coördinate of the first focal point of $H$ in the positive direction from $M$. Let $\left(t_{1}^{\prime}, \cdots, t_{\beta}^{\prime}\right)$ be the $\tau$-coördinates of the first, second, etc., focal points of $H^{\prime}$ which lie in the positive direction from $M^{\prime}$, where focal points are counted according to order.

Let $(t-)$ be the $\tau$-coördinate of an ordinary point of $g$ which lies in the negative direction from $t$, and is very close to $t$, so that no focal points of $H$ or of $H^{\prime}$ lie between $(t-)$ and $t$.

Let $N$ and $h$ be respectively the negative type number and nullity of the invariant function $I(z, w)$ evaluated for $(z)$ taken at the point of $g$ whose $\tau$-coördinate is $(t-)$.

TheOREM 8. In order that the index form $Q(u)$ be positive [or be positive definite] it is both necessary and sufficient that one of the following mutually exclusive weak [or strong] inequalities be satisfied:

$$
t_{1}^{\prime}=t \leqq t_{2}^{\prime}
$$$$
N \quad[\operatorname{or} N+h-1]=0,
$$

$t_{1}^{\prime}<t \leqq t_{2}^{\prime}, \quad N \quad[$ or $N+h-1] \leqq 1$,

$$
\begin{array}{ll}
\dot{(m-1)} & t_{m-2}^{\prime}<t \leqq t_{m-1}^{\prime}, \quad N \quad[\text { or } N+h-1] \leqq m-2, \\
(m) & t_{m-1}^{\prime}<t \leqq t_{m}^{\prime} .
\end{array}
$$$$
t_{2}^{\prime}<t \leqq t_{3}^{\prime}
$$$$
N \cdot[\text { or } N+h-1] \leqq 2 \text {, }
$$

Theorem 8 follows at once from Theorem 7 and Theorem 1, and the proof will be left to the reader.

THEOREM 9. In order that the regular integral $J$ take on a minimum along $g$ it is necessary that one of the mutually exclusive weak inequalities (i), . . ., $(m)$ of Theorem 8 be satisfied.

THEOREM 10. In order that the regular integral $J$ take on a weak proper relative minimum along $g$ it is sufficient that one of the mutually exclusive strong inequalities (i), $\cdots,(m)$ of Theorem 8 be satisfied.

THEOREM 11. In order that the regular integral $J$ take on a minimum along $g$ it is necessary that the inequality

$$
t_{1}^{\prime} \leqq t
$$

be satisfied.

* Bliss (3), loc. cit. 
THEOREM 12. In order that the regular integral $J$ take on a weak proper relative minimum along $g$ it is sufficient that the inequality

$$
t_{m-1}^{\prime}<t
$$

be satisfied.

Theorems 9 and 10 are as close as possible to giving conditions which are both necessary and sufficient. Theorems 11 and 12 (corollaries of Theorems 9 and 10) on the other hand are in general not at all close to giving conditions which are both necessary and sufficient. In order that the index form $Q(u)$ be positive it is not sufficient, in general, that $t_{1}^{\prime} \leqq t$. In order that the index form $Q(u)$ be positive definite it is not in general necessary that $t_{m-1}^{\prime}<t$. In the special case $m=2$, that is, in the problem of the calculus of var ations in 2-space, Theorems 11 and 12 are as close as possible to giving conditions which are both necessary and sufficient. We may state the facts here in the form of two theorems as follows.

THEOREM 13. The classical Jacobi necessary condition in 2-space (that is condition (15.2)) is a sufficient condition in order that the index form be positive, and the classical Jacobi sufficient condition in 2-space (that is condition (15.3) for $m=2$ ) is a necessary condition in order that the index form be positive definite.

THEOREM 14. The generalized necessary Jacobi conditions of Theorem 9 are sufficient conditions in order that the index form be positive and the generalized sufficient Jacobi conditions of Theorem 10 are necessary conditions in order that the index form be positive definite.

Theorems 9, 10, 11, 12, and 14 follow at once from Theorem 8 .

Theorem 13 is somewhat more precise than Theorem 14 for the special case $m=2$. In 2 -space the mutually exclusive conditions of Theorem 8 become the two conditions

(i) $\quad t_{1}^{\prime}=t \leqq t_{2}^{\prime}, \quad N \quad[$ or $N+h-1]=0$,

(ii) $=(m) \quad t_{1}^{\prime}<t \leqq t_{2}^{\prime}$.

It can be shown in 2-space that the strong condition (15.4) (i) is impossible, that is, it never occurs. If $t_{1}^{\prime}=t$ then $h=2$, and the strong condition $N+h-1=0$ cannot be fulfilled. Moreover, if $t_{1}^{\prime}=t$ in 2-space, $h=2$ and $N=0$, so that the weak condition $N=0$ is automatically fulfilled. Hence Theorem 8 states more than is necessary to state in the special case $m=2$. In this case we need merely to state the inequalities between $t_{1}^{\prime}$ and $t$ which occur in the first column of (15.4) without reference to the type numbers of the invariant function. Hence Theorem 13 is true. The proof of this fact requires a slightly 
more extended study of the invariant function.* For the problem of the calculus of variations in space, that is, for $m>2$, we cannot avoid bringing in the type numbers of the invariant function, and for all cases $m>2$, Theorem 8 , and Theorems $9,10,11,12$, and 14 are the most precise theorems which can be stated. They are of course all true for the case $m=2$.

We can also give conditions for a strong minimum as follows.

THEOREM 15. Let $J$ be a regular integral, and let the Weierstrass $E$-function $E(z, r, \sigma)$ be positive for $(z, r)$ taken along the closed segment of $g$ between $M$ and $M^{\prime}$, where $(\sigma)$ is any set not $(0)$ nor proportional to $(r)$. Let one of the mutually exclusive strong inequalities of Theorem 8 be satisfied. Then $J$ takes on a strong proper relative minimum along $g$.

* Cf. Morse (4), loc. cit.

HARVARD UNIVERSITY,

Cambridge, Mass. 